\title{
Inhalts- und Bearbeiterverzeichnis
}

Kaess (Ka), Keukenschrijver (K); Prof. Dr. Maute (M), Prof. Dr. McGuire (MG), Dr. Tochtermann (T), Dr. Werner $(W)$

Hinweise auf Datenbanken und Materialien (K) - XIX

Hinweis zur Zitierweise und zur Nomenklatur - XXII

Allgemeine und abgekürzt zitierte Literatur, Lehrbücher, Kommentare und Einführungen $(\mathrm{K})-\mathbf{X X I I I}$ Abkürzungen - XLI

\section{Patentgesetz}

vom 5.5.1936

in der Fassung der Bekanntmachung vom 16.12.1980

Einleitung $(\mathrm{K})-3$

\section{Erster Abschnitt. Das Patent:}

Vor $§ 1(\mathrm{~K})-39$

$\S 1 \quad$ (Patentfähige Erfindungen) $(\mathrm{K})-\mathbf{4 1}$

§1a (Menschlicher Körper) (K) — 92

$\S 2$ (Vom Patentschutz ausgeschlossene Erfindungen) (K) - 107

$\S 2 \mathrm{a} \quad$ (Pflanzensorten und Tierrassen; Therapie- und Diagnostizierverfahren;

Züchtungsverfahren) (K) - 123

$\S 3 \quad$ (Neuheit) (K) - 146

$\S 4 \quad$ (Erfinderische Tätigkeit) (K) -204

$\S 5$ (Gewerbliche Anwendbarkeit) (K) -280

Vor $\S 6(\mathrm{~K})-284$

$\S 6 \quad$ (Recht auf das Patent) (K) $-\mathbf{2 8 6}$

$\S 7 \quad$ (Formelle Patentberechtigung; Nachanmelderecht) (K) - 307

$\S 8$ (Abtretungs- und Übertragungsanspruch; Klage) (K) - 311

Vor $\S 9(\mathrm{~K})-325$

$\S 9 \quad$ (Wirkung des Patents) (K) - 327

$\S 9 \mathrm{a} \quad$ (Wirkungen bei biologischem Material) (K) - 385

$\S 9 \mathrm{~b} \quad$ (Erschöpfung bei biologischem Material) (K) - 388

$\S 9 \mathrm{c} \quad$ (Landwirteprivileg) (K) - 390

$\S 10$ (Mittelbare Patentverletzung/Patentgefährdung) (W) - 394

$\S 11 \quad$ (Beschränkung der Wirkung des Patents) (K) - 407

$\S 12$ (Vorbenutzung) (K) -422

$\S 13$ (Staatliche Benutzungsanordnung) (K) - 440

$\S 14$ (Schutzbereich) (W) -445

$\S 15$ (Übertragung und Lizenz) $(\mathrm{MG})-490$

$\S 16$ (Patentdauer, Zusatzpatent - weggefallen) $(\mathrm{K})-\mathbf{5 8 0}$

$\S 16 a \quad$ (ergänzendes Schutzzertifikat) $(\mathrm{M})-\mathbf{5 8 3}$

Anhang zu § 16a (M) - 588

$\S 17 \quad$ (Jahresgebühren) (K) - 651

$\S 18$ (Stundung und Erlass von Gebühren, aufgehoben) (K) - 656

$\S 19$ (Vorauszahlung und Rückzahlung von Jahresgebühren, aufgehoben) (K) - 656

$\S 20$ (Erlöschen des Patents) (K) - 656

$\S 21$ (Widerruf des Patents) (K) -662

$\S 22$ (Nichtigkeit des Patents) (K) -687

$\S 23$ (Lizenzbereitschaft) (MG) -691 
$\S 24$ (Zwangslizenz) (MG) -703

Anhang I zu § 24. Die EU-Zwangslizenzverordnung (VO EG Nr 816/2006) (MG) - 731

Anhang II zu § 24. Patentrechtliche Regelungen im EAG-Vertrag, insbesondere Zwangslizenz (K) - 742

$\S 25$ (Inlandsvertreter) (K) -746

\section{Zweiter Abschnitt. Patentamt:}

Vor § 26 Das Europäische Patentamt (K) - 757

$\S 26$ (Deutsches Patent- und Markenamt; Präsident; Mitglieder; Hilfsmitglieder) (K) - 761

$\S 27$ (Prüfungsstellen; Patentabteilungen) (K) -768

Anhang zu § 27. Patentklassifikation (K) -782

$\S 28$ (Organisation; Geschäftsgang des Patentamts; Verordnungsermächtigungen) (K) - 784

$\S 29$ (Gutachten des Patentamts; Auskünfte zum Stand der Technik) (K) -788

§ 29a (Nutzung von urheberrechtlich geschützten Werken) (K) - 792

$\S 30 \quad$ (Patentregister) $(\mathrm{K})-793$

$\S 31 \quad$ (Akteneinsicht) $(\mathrm{K})-\mathbf{8 1 3}$

$\S 31 \mathrm{a} \quad$ Datenschutz $(\mathrm{K})-\mathbf{8 2 9}$

$\S 32$ (Veröffentlichungen des Patentamts) (K) - 830

$\S 33$ (Entschädigungsanspruch nach Offenlegung) (W) - 842

\section{Dritter Abschnitt. Verfahren vor dem Patentamt:}

Vor § 34 (K) - 849

$\S 34$ (Einreichung der Patentanmeldung) (K) - 877

$\S 34 \mathrm{a}$ (Herkunftsangabe bei biologischem Material) (K) - 948

§35 (Anmeldetag; Nachreichung von Zeichnungen und Beschreibungsteilen) (K) - 951

$\S 35 \mathrm{a} \quad$ (Fremdsprachige Unterlagen) $(\mathrm{K})-959$

$\S 36$ (Zusammenfassung) (K) - 963

$\S 37$ (Erfinderbenennung) (K) - 967

$\S 38$ (Änderungen der Anmeldung) (K) -973

$\S 39$ (Teilung der Anmeldung) $(\mathrm{K})-984$

Vor $\S 40(\mathrm{~T})-1010$

$\S 40 \quad$ (Innere Priorität) (T) -1014

$\S 41$ (Ausländische Priorität; Prioritätserklärung) (T) - 1022

Vor $\S 42(\mathrm{~K})-1041$

$\S 42$ (Offensichtlichkeitsprüfung) (K) -1042

$\S 43$ (Recherche) $(\mathrm{K})-\mathbf{1 0 5 2}$

$\S 44 \quad$ (Prüfungsantrag) (K) $-\mathbf{1 0 6 6}$

$\S 45$ (Prüfungsbescheide) (K) $-\mathbf{1 0 7 8}$

$\S 46$ (Sachaufklärung; Anhörung; Niederschrift) (K) - 1090

$\S 47$ (Begründung, Zustellung, Verkündung der Beschlüsse; Rechtsmittelbelehrung) (K) - 1106

$\S 48$ (Zurückweisung der Anmeldung) (K) - 1121

$\S 49$ (Erteilungsbeschluss, Aussetzung der Erteilung) (K) - 1128

$\S 49 \mathrm{a} \quad$ (Prüfung der Anmeldung eines ergänzenden Schutzzertifikats) (M) - 1135

Vor $\S 50(\mathrm{~K})-\mathbf{1 1 4 0}$

$\S 50 \quad$ (Geheimhaltungsanordnung) $(\mathrm{K})-\mathbf{1 1 4 3}$

$\S 51$ (Akteneinsicht für die oberste Bundesbehörde) (K) - 1148

$\S 52$ (Anmeldung im Ausland; Strafvorschrift) (K) - 1149

$\S 53$ (Ausbleiben der Geheimhaltungsanordnung) (K) - 1152

$\S 54 \quad$ (Besonderes Register) $(\mathrm{K})-\mathbf{1 1 5 3}$

$\S 55$ (Entschädigungsanspruch) (K) - 1154

$\S 56$ (Zuständige Behörde) (K) - 1157

$\S 57$ (Erteilungsgebühr, aufgehoben) (K) - 1158 
$\S 58$ (Veröffentlichung der Patenterteilung und der Patentschrift, Eintritt der Patentwirkungen, Rücknahmefiktion) (K) - 1158

$\S 59$ (Einspruch, Beitritt als Einsprechender) (K) - 1163

$\S 60$ (Teilung des Patents im Einspruchsverfahren, aufgehoben) - 1222

$\S 61$ (Entscheidung im Einspruchsverfahren; Veröffentlichung) (K) - 1222

$\S 62$ (Kostenentscheidung; Kostenfestsetzung; Verfahren) (K) $-\mathbf{1 2 4 7}$

$\S 63$ (Erfindernennung) (K) - 1256

$\S 64$ (Widerruf; Patentbeschränkung) (K) - 1263

\section{Vierter Abschnitt. Patentgericht:}

Vor $\S 65(\mathrm{~K})-\mathbf{1 2 7 1}$

$\S 65$ (Bundespatentgericht) (K) - 1272

Anhang zu § 65. Die Beschwerdekammern des Europäischen Patentamts (K) - 1282

$\S 66$ (Senate des Bundespatentgerichts) $(\mathrm{K})-\mathbf{1 2 8 8}$

$\S 67$ (Besetzung der Senate) (K) - 1289

$\S 68$ (Präsidium; Geschäftsverteilung) (K) - 1294

$\S 69$ (Öffentlichkeit; Sitzungspolizei) (K) - 1304

$\S 70 \quad$ (Beratung und Abstimmung) $(\mathrm{K})-\mathbf{1 3 0 8}$

$\S 71 \quad$ (Richter kraft Auftrags; abgeordnete Richter) (K) - 1313

$\S 72 \quad$ (Geschäftsstelle) (K) - 1316

\section{Fünfter Abschnitt. Verfahren vor dem Patentgericht:}

1. Beschwerdeverfahren:

Vor $\S 73(\mathrm{~K})-1321$

$\S 73 \quad$ (Beschwerde) $(\mathrm{K})-\mathbf{1 3 3 8}$

$\S 74 \quad$ (Beschwerderecht) (K) - 1373

$\S 75 \quad$ (Aufschiebende Wirkung) (K) - $\mathbf{1 3 8 2}$

$\S 76 \quad$ (Äußerungsrecht des Präsidenten des Patentamts) (K) - 1385

$\S 77$ (Beitritt des Präsidenten des Patentamts) (K) - 1388

$\S 78 \quad$ (Mündliche Verhandlung) $(\mathrm{K})-\mathbf{1 3 9 0}$

$\S 79$ (Entscheidung) (K) - 1396

$\S 80 \quad$ (Kosten des Beschwerdeverfahrens) (K) - 1415

2. Nichtigkeits- und Zwangslizenzverfahren:

Vor $\S 81(\mathrm{~K})-1442$

$\S 81 \quad$ (Klage) $(\mathrm{K})-\mathbf{1 4 5 1}$

$\S 82$ (Zustellung der Klage; Nichterklärung; Widerspruch; mündliche Verhandlung) (K) - 1481

$\S 83$ (Hinweispflicht; Zurückweisung) (K) - 1525

$\S 84$ (Urteil; Kostenentscheidung) (K) -1534

$\S 85$ (Zwangslizenz: einstweilige Verfügung; vorläufige Vollstreckbarkeit) (K) -1564

$\S 85 a$ (Zwangslizenzen für Patente an der Herstellung pharmazeutischer Erzeugnisse zur Ausfuhr) (K) -1569

3. Gemeinsame Verfahrensvorschriften:

Vor $\S 86(\mathrm{~K})-1571$

$\S 86$ (Ausschließung und Ablehnung) (K) $-\mathbf{1 5 7 1}$

$\S 87$ (Erforschung des Sachverhalts; vorbereitende Anordnungen) (K) -1578

$\S 88$ (Beweiserhebung) (K) - 1584

$\S 89$ (Termin; Ladung) (K) - 1586

$\S 90 \quad$ (Mündliche Verhandlung) $(\mathrm{K})-\mathbf{1 5 8 8}$

$\S 91$ (Erörterung der Sache) (K) 1589

$\S 92 \quad$ (Niederschrift) (K) - 1591 
$\S 93$ (Beweiswürdigung; rechtliches Gehör; Richterwechsel) (K) - 1593

$\S 94$ (Verkündung der Entscheidungen; Begründung) (K) - 1599

$\S 95$ (Berichtigung offenbarer Unrichtigkeiten) (K) $-\mathbf{1 6 0 2}$

$\S 96$ (Tatbestandsberichtigung) $(\mathrm{K})-\mathbf{1 6 0 4}$

$\S 97$ (Vertretung; Vollmacht) (K) $-\mathbf{1 6 0 7}$

$\S 98$ (Auslagen; aufgehoben) $(\mathrm{K})-\mathbf{1 6 1 2}$

§99 (Anwendbarkeit von Gerichtsverfassungsgesetz und Zivilprozessordnung; Anfechtbarkeit; Akteneinsicht) $(\mathrm{K})-1612$

\section{Sechster Abschnitt. Verfahren vor dem Bundesgerichtshof:}

1. Rechtsbeschwerdeverfahren:

Vor $\S 100(\mathrm{~K})-1629$

$\S 100$ (Statthaftigkeit der Rechtsbeschwerde) (K) - 1635

$\S 101$ (Beschwerdeberechtigung; Beschwerdegründe) (K) - 1661

$\S 102$ (Förmliche Voraussetzungen) (K) - 1663

$\S 103$ (Aufschiebende Wirkung) (K) -1667

$\S 104$ (Prüfung der Zulässigkeit) (K) - 1668

$\S 105$ (Mehrere Beteiligte) (K) - 1669

$\S 106$ (Anwendung weiterer Vorschriften) (K) - 1671

$\S 107$ (Entscheidung über die Rechtsbeschwerde) (K) - 1673

$\S 108$ (Zurückverweisung) (K) - 1677

$\S 109$ (Kosten des Rechtsbeschwerdeverfahrens) (K) — 1679

2. Berufungsverfahren:

Vor $\S 110(\mathrm{~K})-1684$

$\S 110 \quad$ (Berufung; Berufungsschrift) $(\mathrm{K})-\mathbf{1 6 8 8}$

$\S 111$ (Berufungsgründe) $(\mathrm{K})-\mathbf{1 6 9 5}$

$\S 112$ (Berufungsbegründung) $(\mathrm{K})-\mathbf{1 6 9 6}$

$\S 113$ (Vertretung) (K) - 1701

$\S 114$ (Prüfung der Zulässigkeit; weiteres Verfahren) (K) — 1703

$\S 115$ (Anschlussberufung) (K) - 1706

$\S 116$ (Prüfungsumfang; Klageänderung) $(\mathrm{K})-\mathbf{1 7 0 8}$

$\S 117$ (Verweisung auf die Zivilprozessordnung) (K) - 1713

$\S 118$ (Mündliche Verhandlung; Ladungsfrist) (K) - 1718

$\S 119$ (Aufhebung und Zurückverweisung; eigene Sachentscheidung) (K) -1719

$\S 120$ (Keine Begründung zu Rügen von Verfahrensmängeln) (K) - 1725

$\S 121$ (Streitwertfestsetzung; Kosten) (K) - 1725

3. Beschwerdeverfahren:

$\S 122$ (Beschwerde gegen Urteile nach $\S \S 85,85 a)(M G)-1731$

4. Gemeinsame Verfahrensvorschriften:

$\S 122 \mathrm{a}$ (Abhilfe bei Verletzung des Anspruchs auf rechtliches Gehör) (K) - 1732

\section{Siebenter Abschnitt. Gemeinsame Vorschriften:}

Vor $\S 123(\mathrm{~K})-\mathbf{1 7 3 9}$

$\S 123$ (Wiedereinsetzung in den vorigen Stand) $(\mathrm{K})-1739$

$\S 123 a$ (Weiterbehandlung) (K) $-\mathbf{1 7 6 9}$

$\S 124$ (Wahrheitspflicht) (K) - 1775

$\S 125$ (Anforderung von Unterlagen) $(\mathrm{K})-\mathbf{1 7 7 9}$

$\S 125$ a (Elektronische Dokumente) $(\mathrm{K})-\mathbf{1 7 8 1}$ 
$\S 126$ (Amtssprache und Gerichtssprache) (K) - 1787

$\S 127$ (Zustellungen) $(\mathrm{K})-\mathbf{1 7 9 4}$

$\S 128$ (Rechtshilfe) (K) - $\mathbf{1 8 0 7}$

$\S 128$ a (Zeugenentschädigung; Sachverständigenvergütung) (K) -1809

$\S 128 \mathrm{~b}$ (Rechtsschutz bei überlangen Gerichtsverfahren) (K) - 1812

\section{Achter Abschnitt. Verfahrenskostenhilfe:}

Vor $\S 129(\mathrm{~K})-1817$

$\S 129$ (Verfahrenskostenhilfe) (K) $-\mathbf{1 8 2 2}$

$\S 130$ (Erteilungsverfahren) (K) - 1823

$\S 131$ (Widerrufs- und Beschränkungsverfahren) (K) - 1836

$\S 132$ (Einspruchs- und Nichtigkeitsverfahren) (K) - 1837

$\S 133$ (Beiordnung eines Vertreters) $(\mathrm{K})-\mathbf{1 8 4 2}$

$\S 134$ (Hemmung von Fristen) (K) 1848

$\S 135$ (Bewilligungsverfahren) $(\mathrm{K})-\mathbf{1 8 5 0}$

$\S 136$ (Anwendbarkeit von Bestimmungen der Zivilprozessordnung) (K) $-\mathbf{1 8 5 5}$

$\S 137$ (Aufhebung) (K) - 1858

$\S 138$ (Verfahrenskostenhilfe im Rechtsbeschwerdeverfahren) (K) - 1860

\section{Neunter Abschnitt. Rechtsverletzungen:}

Vor $\S 139(\mathrm{~W}) \longrightarrow \mathbf{1 8 6 3}$

$\S 139$ (Unterlassungsanspruch; Schadensersatz) (W) - 1866

$\S 140$ (Aussetzung des Rechtsstreits bei Ansprüchen aus offengelegter Anmeldung) (Ka) - 1957

$\S 140 a$ (Vernichtungs- und Rückrufanspruch) (Ka) - 1968

$\S 140 b$ (Auskunft über Herkunft und Vertriebsweg) (Ka) - 1979

$\S 140 \mathrm{c}$ (Vorlage und Besichtigung) (Ka) - 2004

$\S 140 d$ (Vorlage von Bankunterlagen) (Ka) - 2022

$\S 140$ e (Urteilsbekanntmachung) (Ka) -2026

$\S 141$ (Verjährung) (K) 2032

$\S 141 \mathrm{a}$ (Andere Anspruchsgrundlagen) (K) -2042

$\S 142$ (Strafbestimmung; strafrechtliche Einziehung; Veröffentlichungsbefugnis) (K) - 2058

§ 142a (Beschlagnahme durch die Zollbehörde) (M) - 2071

$\S 142 b$ (Verfahren nach der Grenzbeschlagnahmeverordnung) (M) - 2083

\section{Zehnter Abschnitt. Verfahren in Patentstreitsachen:}

Vor $§ 143(\mathrm{Ka})-2091$

$\S 143$ (Patentstreitsachen) (Ka) -2198

$\S 144$ (Streitwertherabsetzung) (K) -2236

$\S 145$ (Klagenkonzentration) (K) -2242

$\S 145 \mathrm{a}$ DiskE (Schutz von Geschäftsgeheimnissen) (K/Ka/MG/W) — 2249

\section{Elfter Abschnitt. Patentberühmung:}

$\S 146$ (Auskunftsanspruch) (K) 2255

Zwölfter Abschnitt. Übergangsvorschriften:

Vor $\S 147(\mathrm{~K})-2263$

$\S 147$ (Übergangsbestimmungen) $(\mathrm{K})-2265$ 


\section{Patentkostengesetz}

vom 13.12.2001

(K)

Einleitung PatKostG -2272

$\S 1 \quad$ Geltungsbereich, Verordnungsermächtigungen - 2280

$\S 2$ Höhe der Gebühren — 2281

§3 Fälligkeit der Gebühren -2283

$\S 4 \quad$ Kostenschuldner -2285

$\S 5 \quad$ Vorauszahlung, Vorschuss - 2287

§ 6 Zahlungsfristen, Folgen der Nichtzahlung - 2290

$\S 7$ Zahlungsfristen für Jahres-, Aufrechterhaltungs- und Schutzrechtsverlängerungsgebühren, Verspätungszuschlag - 2293

$\S 8 \quad$ Kostenansatz - 2295

§ 9 Unrichtige Sachbehandlung - 2296

$\S 10$ Rückzahlung von Kosten, Wegfall der Gebühr - 2297

$\S 11 \quad$ Erinnerung, Beschwerde -2302

$\S 12$ Verjährung, Verzinsung - $\mathbf{2 3 0 4}$

$\S 13$ Anwendung der bisherigen Gebührensätze - 2306

$\S 14$ Übergangsvorschrift aus Anlass des Inkrafttretens dieses Gesetzes - 2308

$\S 15$ Übergangsvorschriften aus Anlass des Inkrafttretens des Geschmacksmuster-

reformgesetzes -2309

\section{Gebrauchsmustergesetz}

vom 5.5.1936

in der Fassung der Bekanntmachung vom 28.8.1986

(K)

Einleitung GebrMG — 2312

$\S 1 \quad$ (Gebrauchsmusterfähigkeit) -2317

$\S 2$ (Schutzausschlüsse) -2323

$\S 3$ (Neuheit; gewerbliche Anwendbarkeit) - 2325

$\S 4$ (Anmeldung; Änderungen; Teilung) - 2329

$\S 4 \mathrm{a}$ (Anmeldetag; Nachreichung von Zeichnungen und Beschreibungsteilen) - 2335

$\S 4 \mathrm{~b} \quad$ (Fremdsprachige Unterlagen) -2336

$\S 5$ (Abzweigung) -2337

$\S 6 \quad$ (Priorität) -2343

$\S 6 \mathrm{a} \quad$ (Ausstellungsschutz) -2345

$\S 7 \quad$ (Recherche) - 2347

$\S 8 \quad$ (Eintragung; Register) - 2352

$\S 9 \quad$ (Geheimgebrauchsmuster) -2358

$\S 10$ (Gebrauchsmusterstelle; Gebrauchsmusterabteilungen; Verordnungsermächtigung) $-\mathbf{2 3 5 9}$

$\S 11 \quad$ (Wirkung der Eintragung) -2362

$\S 12$ (Beschränkung der Wirkung) 2363

$\S 12 \mathrm{a} \quad$ (Schutzbereich) -2364

$\S 13$ (Nichteintritt des Schutzes; Entnahme; Verweisungen) - 2366

$\S 14$ (Gebrauchsmuster und jüngeres Patent) - 2368

Vor $\S 15-2370$

$\S 15$ (Löschungsgründe; Antragsberechtigung; Teillöschung) - 2374

$\S 16$ (Löschungsantrag) - 2379

$\S 17$ (Löschungsverfahren) - 2384

$\S 18$ (Beschwerde; Rechtsbeschwerde) -2399 
$\S 19$ (Aussetzung des Verletzungsstreits) $-\mathbf{2 4 0 7}$

$\S 20$ (Gebrauchsmusterzwangslizenz) - 2411

$\S 21$ (Verweisungen auf das Patentgesetz) -2412

$\S 22$ (Übertragung und Lizenz) - 2414

$\S 23$ (Schutzdauer; Aufrechterhaltung; Erlöschen) - 2415

$\S 24$ (Unterlassungsanspruch; Schadensersatz) - 2419

§ 24a Zivilrechtlicher Vernichtungsanspruch) - 2422

$\S 24 \mathrm{~b}$ (Auskunft über Herkunft und Vertriebsweg) -2423

$\S 24 \mathrm{c} \quad$ (Vorlage und Besichtigung) -2424

$\S 24 \mathrm{~d}$ (Vorlage von Unterlagen) - 2425

$\S 24 \mathrm{e}$ (Urteilsbekanntmachung) - 2426

$\S 24 \mathrm{f}$ (Verjährung) - 2426

$\S 24 \mathrm{~g}$ (Andere Anspruchsgrundlagen) - 2426

$\S 25$ (Strafbestimmung; strafrechtliche Einziehung; Veröffentlichungsbefugnis) $-\mathbf{2 4 2 7}$

$\S 25 a \quad$ (Beschlagnahme durch die Zollbehörde) - 2428

$\S 25 \mathrm{~b} \quad$ (Verfahren nach der Grenzbeschlagnahmeverordung) — 2429

$\S 26$ (Teilstreitwert) $\mathbf{2 4 2 9}$

Vor $\S 27$ (Verfahren in Gebrauchsmusterstreitsachen) - $\mathbf{2 4 3 0}$

$\S 27$ (Gebrauchsmusterstreitsachen) - 2431

$\S 28$ (Inlandsvertreter) - $\mathbf{2 4 3 2}$

$\S 29$ (Verordnungsermächtigung) -2433

$\S 30$ (Auskunftsanspruch) - $\mathbf{2 4 3 4}$

$\S 31$ (Übergangsbestimmung) 2435

\section{Gesetz über den Schutz der Topographien von elektronischen Halbleitererzeugnissen}

vom 22.10.1987

(K)

Einleitung HISchG -2437

\section{Erster Abschnitt. Der Schutz der Topographien:}

$\S 1 \quad$ Schutzgegenstand, Eigenart - 2441

$\S 2$ Recht auf den Schutz der Topographie - 2443

§3 Anmeldung - 2445

§4 Eintragung, Bekanntmachung, Änderungen, Akteneinsicht, Topographiestelle, Topographieabteilung - $\mathbf{2 4 4 7}$

$\S 5 \quad$ Entstehung des Schutzes, Schutzdauer $-\mathbf{2 4 5 0}$

$\S 6 \quad$ Wirkung des Schutzes - 2451

$\S 7 \quad$ Beschränkung der Wirkung des Schutzes - 2453

$\S 8 \quad$ Löschungsanspruch, Löschungsverfahren -2454

$\S 9 \quad$ Schutzverletzung -2455

$\S 10$ Strafvorschriften $-\mathbf{2 4 5 5}$

$\S 11$ Anwendung von Vorschriften des Patentgesetzes und des Gebrauchsmustergesetzes - 2456

Zweiter Abschnitt. Änderung von Gesetzen auf dem Gebiet des gewerblichen Rechtsschutzes:

$\S \S 12-16$ (nicht abgedruckt) -2457 
Dritter Abschnitt. Änderung anderer Gesetze:

$\S \S 17-25$ (nicht abgedruckt) -2457

Vierter Abschnitt. Übergangs- und Schlußvorschriften:

$\S 26$ Übergangsvorschriften $-\mathbf{2 4 5 8}$

$\S 27$ Berlin-Klausel -2458

$\S 28$ Inkrafttreten -2458

\section{Gesetz über Arbeitnehmererfindungen}

vom 25.7.1957

(K)

Einleitung ArbEG $\longrightarrow \mathbf{2 4 5 9}$

Erster Abschnitt. Anwendungsbereich und Begriffsbestimmungen:

$\S 1 \quad$ Anwendungsbereich -2465

$\S 2$ Erfindungen $-\mathbf{2 4 6 8}$

§3 Technische Verbesserungsvorschläge - 2470

$\S 4$ Diensterfindungen und freie Erfindungen - 2471

Zweiter Abschnitt. Erfindungen und technische Verbesserungsvorschläge von Arbeitnehmern im privaten Dienst:

1. Diensterfindungen:

Vor $\S 5-2474$

$\S 5 \quad$ Meldepflicht -2474

$\S 6 \quad$ Inanspruchnahme $-\mathbf{2 4 8 1}$

$\S 7 \quad$ Wirkung der Inanspruchnahme -2485

$\S 8 \quad$ Frei gewordene Diensterfindungen $-\mathbf{2 4 8 7}$

Vor $\S 9-2490$

$\S 9$ Vergütung bei Inanspruchnahme - 2491

$\S 10$ Vergütung bei beschränkter Inanspruchnahme - 2503

$\S 11 \quad$ Vergütungsrichtlinien -2503

$\S 12$ Feststellung und Festsetzung der Vergütung - 2525

$\S 13$ Schutzrechtsanmeldung im Inland - 2541

$\S 14$ Schutzrechtsanmeldung im Ausland - 2547

§15 Gegenseitige Rechte und Pflichten beim Erwerb von Schutzrechten - 2553

$\S 16$ Aufgabe der Schutzrechtsanmeldung oder des Schutzrechts - 2556

$\S 17$ Betriebsgeheimnisse $-\mathbf{2 5 6 5}$

2. Freie Erfindungen:

Vor $\S 18-2568$

$\S 18$ Mitteilungspflicht $-\mathbf{2 5 6 8}$

$\S 19$ Anbietungspflicht $-\mathbf{2 5 7 1}$

3. Technische Verbesserungsvorschläge:

$\S 20-2574$ 
4. Gemeinsame Bestimmungen:

$\S 21$ (aufgehoben) - 2579

$\S 22$ Unabdingbarkeit $-\mathbf{2 5 8 0}$

$\S 23$ Unbilligkeit - 2582

$\S 24$ Geheimhaltungspflicht -2586

$\S 25$ Verpflichtungen aus dem Arbeitsverhältnis - 2588

$\S 26$ Auflösung des Arbeitsverhältnisses - 2589

$\S 27$ Insolvenzverfahren $-\mathbf{2 5 9 0}$

5. Schiedsverfahren:

Vor $\S 28-2593$

$\S 28$ Gütliche Einigung - 2594

§ 29 Errichtung der Schiedsstelle - 2597

$\S 30$ Besetzung der Schiedsstelle - 2598

$\S 31$ Anrufung der Schiedsstelle $-\mathbf{2 6 0 0}$

§32 Antrag auf Erweiterung der Schiedsstelle - 2601

§33 Verfahren vor der Schiedsstelle - 2601

$\S 34$ Einigungsvorschlag der Schiedsstelle - 2604

$\S 35$ Erfolglose Beendigung des Schiedsverfahrens - 2608

$\S 36$ Kosten des Schiedsverfahrens - 2609

6. Gerichtliches Verfahren:

Vor $\S 37-\mathbf{2 6 1 0}$

$\S 37$ Voraussetzungen für die Erhebung der Klage - 2610

$\S 38$ Klage auf angemessene Vergütung - 2612

$\S 39$ Zuständigkeit — 2613

Dritter Abschnitt. Erfindungen und technische Verbesserungsvorschläge von Arbeitnehmern im öffentlichen Dienst, von Beamten und Soldaten:

$\S 40$ Arbeitnehmer im öffentlichen Dienst - 2617

$\S 41$ Beamte, Soldaten - 2619

$\S 42$ Besondere Bestimmungen für Erfindungen an Hochschulen - 2619

Vierter Abschnitt. Übergangs- und Schlussbestimmungen:

$\S 43$ Übergangsvorschrift - 2628

$\S 44$ (aufgehoben) - 2630

$\S 45$ Durchführungsbestimmungen - 2630

$\S 46$ Außerkrafttreten von Vorschriften - 2631

$\S 47$ (aufgehoben) - $\mathbf{2 6 3 1}$

$\S 48$ (aufgehoben) - 2631

$\S 49$ Inkrafttreten - 2631 


\title{
Gesetz über internationale Patentübereinkommen
}

\author{
vom 21.6.1976
}

(K)

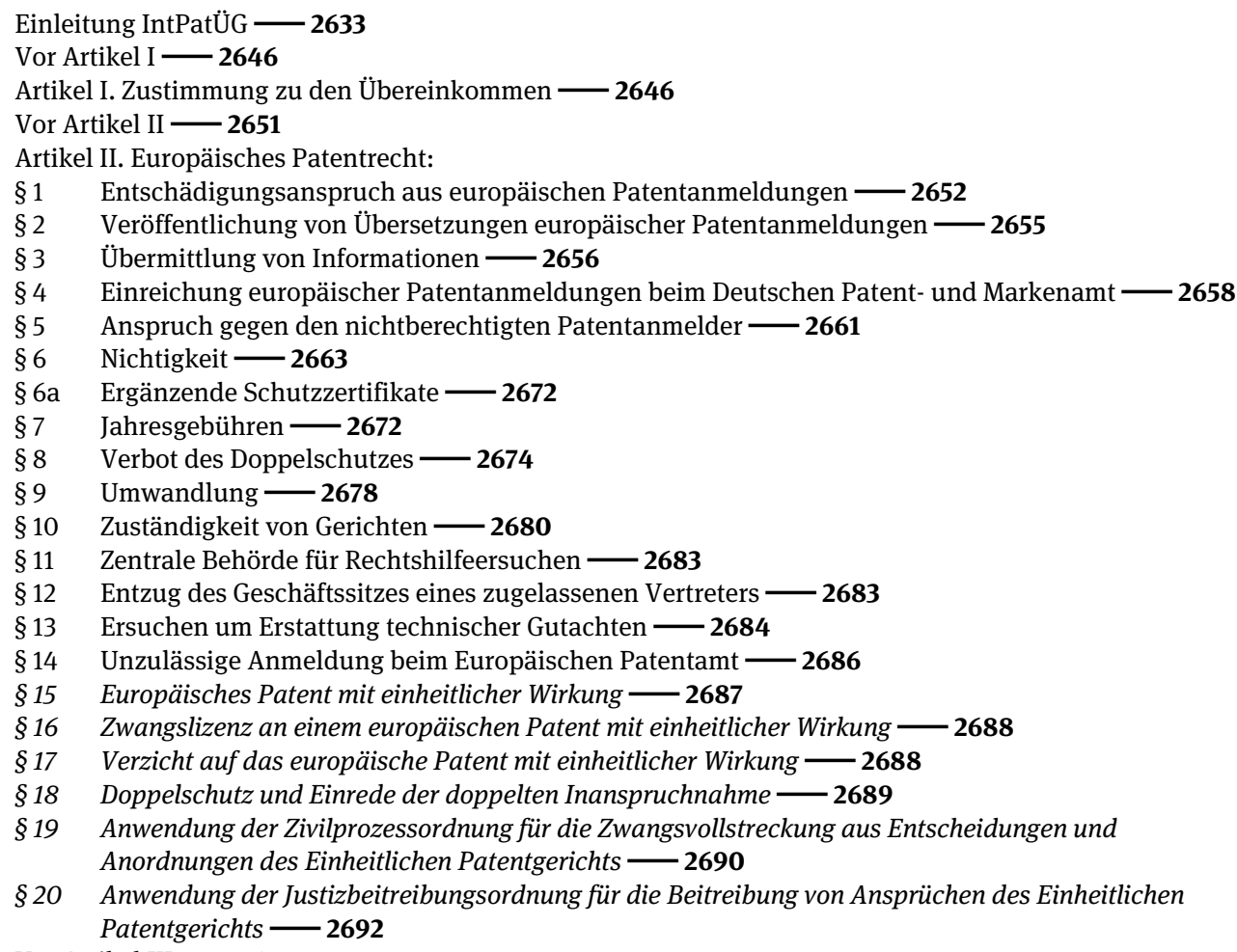

Vor Artikel III — 2693

Artikel III. Verfahren nach dem Patentzusammenarbeitsvertrag:

$\S 1$ Das Deutsche Patent- und Markenamt als Anmeldeamt - 2698

$\S 2$ Geheimhaltungsbedürftige internationale Anmeldungen - 2702

§3 Internationale Recherchenbehörde - 2703

$\S 4$ Das Deutsche Patent- und Markenamt als Bestimmungsamt - 2704

§ 5 Weiterbehandlung als nationale Anmeldung - 2709

§ 6 Das Deutsche Patent- und Markenamt als ausgewähltes Amt - 2710

§ 7 Internationaler Recherchenbericht - $\mathbf{2 7 1 2}$

$\S 8 \quad$ Veröffentlichung der internationalen Anmeldung - 2712

Artikel IV, V, VI (nicht abgedruckt) - 2713-2714

Artikel VII. Einschränkung von Vorschriften der Patentanwaltsordnung und der Bundesrechtsanwaltsordnung -2714

Artikel VIII, IX (nicht abgedruckt) -2714

Artikel X. Bekanntmachung von Änderungen - 2714

Artikel XI. Übergangs- und Schlußbestimmungen:

$\S 1$ Geltungsbereich des Art. IV IntPatÜG 2715

$\S 2$ (nicht abgedruckt) -2715

§3 (nicht abgedruckt) $-\mathbf{2 7 1 5}$

$\S 4$ Geltungsbereich des Übersetzungserfordernisses für europäische Patente - 2716

$\S 5$ Geltungsbereich der durch das Gesetz zur Anpassung patentrechtlicher Vorschriften auf Grund der europäischen Patentreform geänderten Regelungen - $\mathbf{2 7 2 0}$ 


\section{Einheitlicher Patentschutz in Europa}

(K) -2723

\section{Anhang}

Entscheidungs- und Fundstellenregister BGH $\mathbf{- 2 7 3 1}$

Sachregister $-\mathbf{2 8 3 1}$ 
